\section{Evaluation of oral hygiene serv- ices in selected public health facilities in Lusaka district of Zambia}

\author{
Shary Josephin, ${ }^{1}$ Selestine Nzala, ${ }^{1}$ \\ Kumar S. Baboo ${ }^{2}$ \\ ${ }^{1}$ Department of Health Policy and \\ Management, School of Public Health, \\ University of Zambia; ${ }^{2}$ School of Public \\ Health, University of Zambia, Lusaka, \\ Zambia
}

\begin{abstract}
Around the globe many people are suffering from oral pain and other problems of the mouth or teeth. This public health problem is growing rapidly in developing countries where oral health services are limited. This study was conducted in the public health centers and first level hospitals with dental units (DUs) in Lusaka district. Adequacy of dental equipment, instruments and materials required for scaling and polishing (S\&P) and oral hygiene instructions (OHI) was evaluated. Challenges faced by the dental therapists (DTs) in the entire DUs were also evaluated. This was a mixed method study; quantitative data was collected using a structured questionnaire which contained a standard facility assessment check list. Qualitative data was collected by conducting in-depth interviews. The study results showed that the oral hygiene services offered by the selected DUs in Lusaka district were inadequate. A fully functional dental chair was available only in fifty percent of the DUs, only twenty percent of the facilities had fully functional ultrasonic scaling unit, none of the facilities had all the instruments for polishing. The DTs work under stressful conditions as sixty percent of the facilities were understaffed; the supply of dental instruments and materials was poor; delay in repairing faulty equipment; risk of exposure to air borne infections as most of the facilities have space and ventilation problems. Recommendations of this study point towards an urgent need to improve and coordinate the oral health care system.
\end{abstract}

\section{Introduction}

The public health problems associated with oral disease are a serious burden on countries around the globe. ${ }^{1}$ According to the Ministry of Health Zambia, though most of the oral diseases are preventable, over
$80 \%$ of Zambians are affected by oral health problems, which include dental caries, periodontal disease, malocclusion, facial injuries, halitosis (bad breath), cancrum oris, and oral tumors. The scourge of HIV \& AIDS has also compounded the deleterious impact of oral diseases in the Zambian population. In Zambia currently, no comprehensive epidemiological studies have been done so far to ascertain the extent of NCDs in the population, but anecdotal data indicate that NCDs are an emerging problem and requires urgent attention. Oral diseases are common in Zambia, and there has been an insufficient focus on preventive strategies. ${ }^{2}$ All health facilities providing dental services must have dental equipment, medicines and other allied products available in the clinic. It must be suitable for the scope of services and should be operated by qualified staff. ${ }^{3}$ Thus it is essential that dental equipment and instruments should be available, functional and adequate and dental materials must be available and adequate in all dental facilities in order to provide the necessary treatments. This study focusses on oral prophylactic treatment - S\&P and OHI. It is also vital to know the working environment in which these services are delivered and also whether the dental professionals face any difficulties in providing dental care services.

\section{Importance of oral hygiene in sys- temic diseases}

Sub-Saharan Africa remains the region most heavily affected by HIV. In 2008, SubSaharan Africa accounted for $67 \%$ of HIV infections worldwide and $68 \%$ of HIV infections among children. The region also accounted for $72 \%$ of the world's AIDSrelated deaths in $2008 .^{4}$

Subjects with periodontal diseases have higher odds and higher risks of developing cardiovascular diseases but the reduction in the risk of cardiovascular events associated with the treatment of periodontitis remains to be investigated. ${ }^{5}$

Periodontal disease and tooth loss are linked to chronic diseases such as diabetes mellitus; the growing incidence of diabetes in several countries may therefore have a negative impact on oral health. ${ }^{6}$

The results of a cross-sectional study suggest that male chronic obstructive pulmonary disease (COPD) may be associated with the severe periodontitis and indicate the importance of promoting dental care in COPD patients. ${ }^{7}$

Scaling is the mechanical removal of both sub gingival and supra gingival deposits on tooth surface. This treatment combined with maintenance of oral hygiene helps to prevent early stages of gum disease
Correspondence: Shary Josephin, Department of Health Policy and Management, School of Public Health, University of Zambia, Post Box -50110, Lusaka, Zambia.

Tel.: +260976149523.

E-mail: sharysony@hotmail.com

Acknowledgements: the authors are grateful to Mrs Doreen Mwondela, Provincial oral health coordinator, Ministry of Health, Zambia and Dr Prasanth Thankian, University of Zambia, for their guidance.

Key words: Oral hygiene; developing countries; ultrasonic; dental equipment; health facilities.

Contributions: SJ, conception of the work, data collection, data analysis and interpretation, drafting, revising, manuscript writing. $\mathrm{SN}$, supervision, revising, approval of work $\mathrm{KB}$, supervision, revising, approval of work.

Conflict of interests: the authors declare no potential conflict of interest.

Received for publication: 16 November 2017 Revision received: 20 June 2018.

Accepted for publication: 5 July 2018.

This work is licensed under a Creative Commons Attribution NonCommercial 4.0 License (CC BY-NC 4.0).

CCopyright S. Josephin et al., 2018

Licensee PAGEPress, Italy

Journal of Public Health in Africa 2018; 9:820 doi:10.4081/jphia.2018.820

from progressing to more severe forms.

S\&P should always be followed by $\mathrm{OHI}$ so as to help the patient to maintain oral health.

OHI help to educate the patient on maintenance of oral hygiene, which will prevent oral diseases from recurring. Chairside oral health promotion has been shown to be effective more consistently than any other methods of oral health promotion. ${ }^{8}$

A repeated, thorough oral hygiene instruction can achieve a similar benefit to repeated professional mechanical plaque removal. ${ }^{9}$

\section{Human resource for oral health services}

In the face of increased population and increased disease burden, there are very few health workers in the Zambian public sector. They are overwhelmed, demotivated and underpaid. Corrective measures have been started but these need to be strengthened and as comprehensive as can be. ${ }^{10}$

The staffing of the DUs comprises of DTs and dental assistants. There is a need to 
look into the staffing of the DUs i.e. whether there are enough DTs that can cater to the needs of the patients attending these DUs. It is also necessary to look into their working environment and the difficulties they face while providing dental care services.

\section{Materials and Methods}

\section{Study design}

This was a mixed method study, a convergent design. A descriptive cross-sectional survey was carried out to evaluate the adequacy of oral hygiene services. The quantitative and qualitative strands were independent at data collection and analysis but were merged at interpretation of findings.

\section{Study site}

This study was conducted in Lusaka district. The urban health centers or clinics serve a catchment population of between 30,000 to 50,000 people and First level hospitals, also referred to as District Hospitals; serve a population of between 20,000 and 80,000 . There are twenty-five public health centres and eight first level hospitals in Lusaka District. Five first level hospitals and five health centres have DUs. Study was conducted in all the ten DUs, staffing of each dental unit consists of DTs and an assistant (not professionally trained) Primary dental care services are provided to the public from these clinics.

\section{Study population}

The study population for the quantitative strand was ten DUs - five health centres and five first level hospitals one DT form each facility was chosen to fill in the facility assessment check list. For the qualitative strand, the study population was ten DTs working in each of the DUs.

\section{Determination of sample size Quantitative strand}

The study sampled all the ten dental clinics and one DT was purposively sampled from each dental clinic to fill in the facility assessment checklist.

\section{Qualitative strand}

The study was done by purposively sampling one DT from each of the ten clinics according to seniority of service for indepth interviews.

\section{Data collection method Quantitative data}

Quantitative data in this study was col- lected using a structured questionnaire, which was administered to DTs. No study tool was available to be used in this study; therefore a questionnaire that contained a facility assessment checklist was made. The facility assessment checklist questionnaire had three dimensions of oral hygiene services which include;

Availability, functionality and adequacy of: Dental equipment and instruments required for S\&P: i) Essential equipment: - dental chair, dental light, dentist stool, dental compressor, suction lines, bench top sterilizing unit; ii) Diagnostic dental instruments: - dental tray, mouth mirrors, explorers; iii) Instruments for S\&P: - ultrasonic scaler unit, ultrasonic scaler tips, manual scaling set, suction tips, prophylaxis hand piece, prophylaxis angle, prophylaxis cup/brush; iv) Personal protective equipment: - surgical gloves, mouth mask, protective eye wear.

Availability and adequacy of: Essential dental materials for polishing: - prophylaxis paste.

- OHI to patients: - instruct to brush with tooth paste twice daily using a soft bristle brush, brushing technique demonstration, instruct to change toothbrush every three months, use of dental floss demonstration, instruct to use dental floss and mouth wash, dietary instructions.

A score of either one or zero was given on the questionnaire depending on whether the particular equipment, instrument or material etc. is available or not available, functional or non-functional, adequate or inadequate. Adequacy of a particular equipment, instrument or material was calculated depending on whether the DTs were able to provide treatment with these to majority of the patients coming to the DUs.

\section{Qualitative data}

Qualitative data was collected by an indepth interview using an interview guide that was administered to the DTs to determine the challenges faced by the DTs in administering dental care services to patients; audio recordings were done. Pretest of the instrument was conducted with two DTs one working in a dental unit in a health centre and the other working in a first level hospital in order to determine whether the respondents were bringing out the required response. All the ten DTs participated in the study.

\section{Data analysis Quantitative data}

Data was compiled manually on an excel spreadsheet and entered in SPSS.
Descriptive statistical analyses were performed using the different dimension scores in the oral hygiene checklist in order to obtain a clear understanding of the population. Measures of central tendency were computed. Data was summarized and presented graphically to describe the sample.

\section{Qualitative data}

Data was collected as audio recordings. It was transcribed as textual files manually and NVIVO tests were used to assist in the coding and data management. Relevant words, phrases or sentences were coded and categories were created using several codes. Categories were labelled and decisions were made about which are the most relevant and how they are connected to each other; the connections were described, which is the result of the study.

Qualitative and quantitative data was analysed separately and then merged. Then qualitative data was used to build on the quantitative data to make conclusions and interpretations.

\section{Results}

\section{Availability, functionality and ade-} quacy of essential equipment

Figure 1 shows the availability and functionality of essential dental equipment in the entire DUs, though most of the essential equipment is available, not all of them are functional. Only $50 \%$ of the facilities have a fully functional dental chair.

\section{Availability, functionality and ade- quacy of diagnostic dental instru- ments}

All of these instruments, particularly the mouth mirrors (20\% adequacy) were found to be inadequate (Figure 2). $80 \%$ of the facilities had broken or blurred mouth mirrors. Since the DTs in these facilities are not provided with clear and adequate mouth mirrors, they don't use mouth mirrors for examination.

\section{Availability, functionality and ade- quacy of instruments for scaling and polishing \\ Only $20 \%$ of the clinics have a fully} functional ultrasonic scaling unit and ultrasonic scaling tips which are used for scaling and only $10 \%$ of the clinics have functional prophylaxis angle and none of the clinics have prophylaxis cup/brush (Figure 3).

Availability and adequacy of personal protective equipment

Surgical gloves are available and ade- 
quate in all the clinics but only $80 \%$ of the clinics have adequate mouth masks. Only $30 \%$ of the clinics have adequate protective eye wear (Figure 4).

\section{Availability and adequacy of essen- tial material for polishing}

The Polishing material is available in only $20 \%$ of the facilities and only $10 \%$ have adequate polishing material. But polishing is not done because they do not have a prophylaxis brush/cup.

\section{Oral hygiene instructions}

Figure 5 indicates the $\mathrm{OHI}$ given to patients. Instructions of using dental floss, mouth wash and dietary instructions are mostly not given to the patients. Providing $\mathrm{OHI}$ to all patients is not given importance in these clinics. OHI are given to only a selected few patients due to time limitations. $70 \%$ of the dental professionals do not advise the patients about use of dental floss and mouthwash. The working environment in the DUs makes it difficult to give $\mathrm{OHI}$ to patients. None of the facilities provide all the patients with one on one $\mathrm{OHI}$. According to all the DTs, providing one on one $\mathrm{OHI}$ to all the patients will only be possible if the clinics have at least two dental chairs and two DTs.

This section describes the major work related challenges.

The DTs undergo work related stress. Their work does not meet their expectations at all and were much disappointed.

\section{Non-functional and inadequate den- tal chair}

Providing dental treatment using a nonfunctional dental chair is extremely difficult. Some facilities have a completely nonfunctional dental chair, there is no spittoon attached to the dental chair, the patients coming to these facilities have to either spit into the wash basin, or have to spit into the waste bin during treatment. Since the therapists have no choice but to give treatment, they somehow improvise.

\section{Lack of instruments and materials}

In most of the clinics the ultrasonic scaler stopped working years back. Patients requiring $\mathrm{S} \& \mathrm{P}$ are referred to higher facilities. Despite being rusted, some of the instruments for dental extraction are still being used. They do not have the required instruments for extraction also.

\section{Delay in repairing equipment}

Dental equipment needs servicing and repair due to wear and tear. Nine out of the ten DTs interviewed said that they have to wait for a very long time for the technician to do the servicing and repair of equipment.

\section{Poor staffing}

A dental unit requires a minimum of two DTs as one personnel cannot manage all the patients alone. $60 \%$ of the facilities are understaffed; the therapists working in these facilities said that their job is very hectic. The DTs in these facilities are unable to give OHI to all patients because of lack of time as they have to handle the facility singlehandedly.

\section{Risk of infections and overall health issues}

$60 \%$ of DUs has space and ventilation problems. In some clinics the ventilation is poor because the windows cannot be opened. There is a risk of spread of airborne infections. Working with a non-functional dental chair leads to musculoskeletal issues such as neck and back pain.

\section{Challenges after the removal of user fee}

User fee that was charged sometime back was a help for the facilities, the money collected was used to buy the necessary materials, but now the services are free of charge. They have to wait for a long time till the government supplies them.

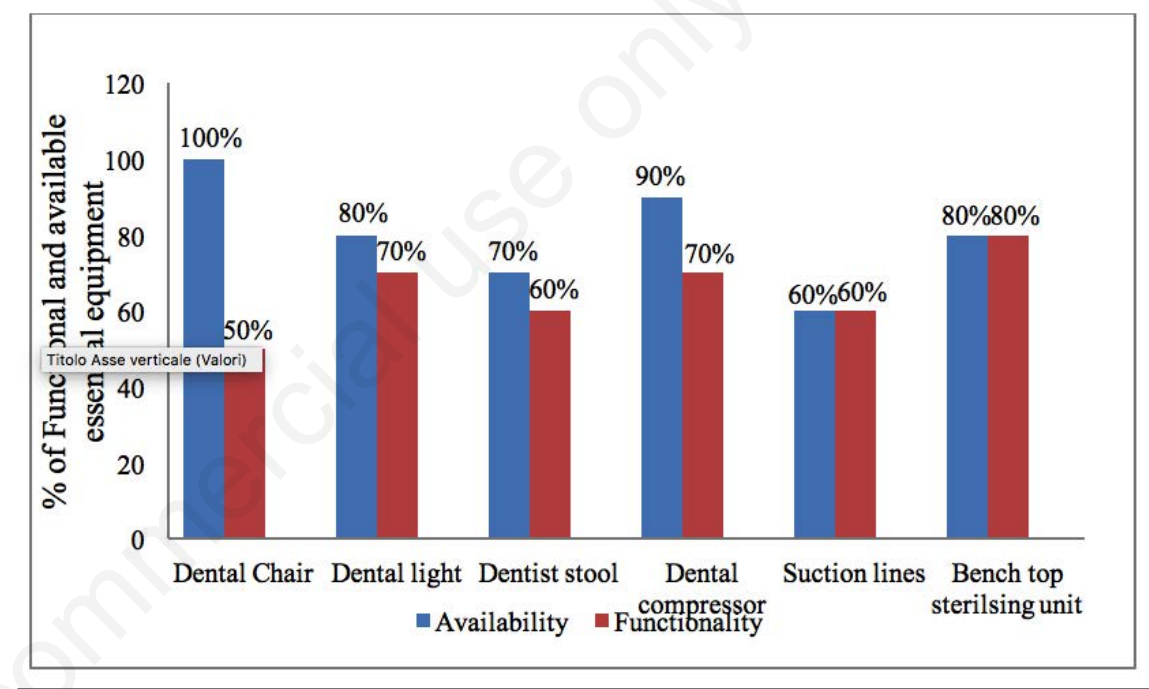

Figure 1. Availability and functionality of essential equipment.

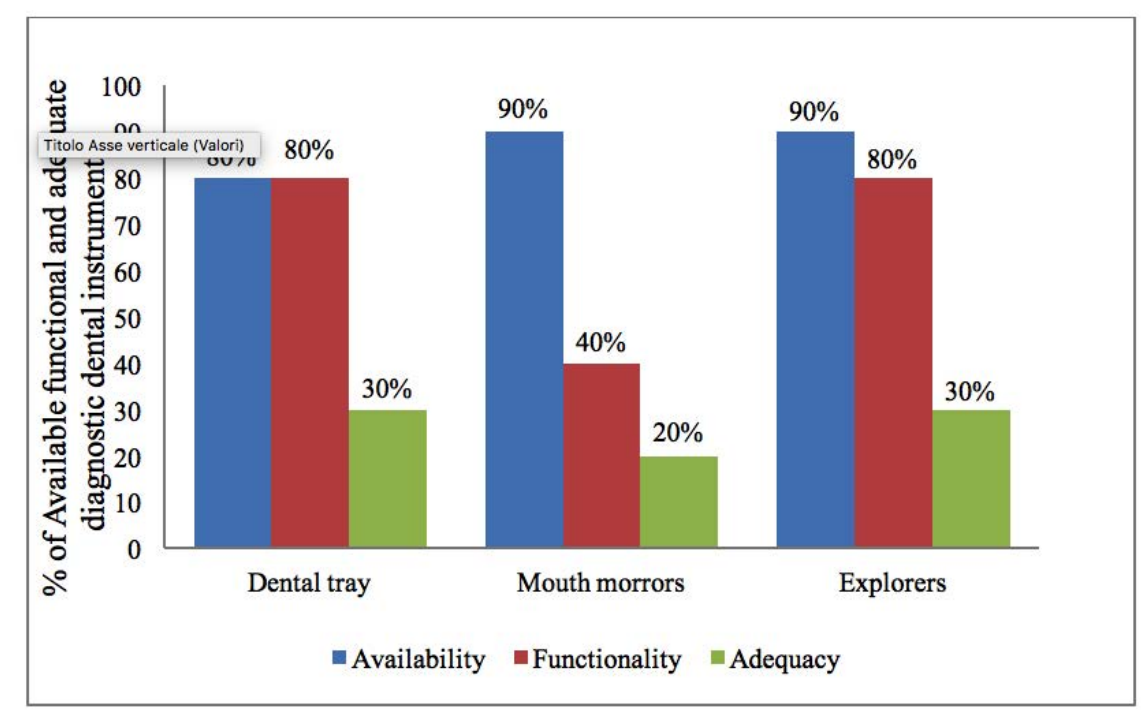

Figure 2. Availability functionality and adequacy of diagnostic dental instruments. 


\section{Discussion}

The study results highlight that only $20 \%$ of the DUs have all the equipment and instruments available and functional for scaling and none of the DUs have all the instruments available for polishing. In terms of adequacy none of them have adequate equipment and instruments to provide scaling to all the patients who require scaling. Though most of the essential equipment and diagnostic instruments are available, majority of them are not functional and none are adequate to meet the requirements of the population. In contrast to this, a study done in Tanzania showed that scaling was provided by $86 \%$ of the public health facilities. ${ }^{11}$ Results of a similar study done in India showed that the availability of dental equipment and dental services were not streamlined. ${ }^{12}$

There is a great need to look into the supply of adequate instruments for scaling. However the manual scaling set is hardly used by the DTs as manual scaling is quiet time consuming, which is in line with a study done in India. The study showed that dental care units having no ultrasonic scaler relied on option of hand scaling which indeed is a tiring and time consuming procedure, thus affecting the efficiency of dental manpower. ${ }^{13}$

The results of this study indicate that only $30 \%$ of the clinics have adequate protective eye wear. In a study that looked at the prevalence of ocular injury and the use of protective eye wear among the dental personnel, found that the frequency of using protective eye wear among the dental personnel of Lagos University Teaching hospital was also found to be low. The use of protective eye wear should be emphasized for all dental personnel and patients to prevent the occurrence of ocular injuries in the clinic and laboratory. ${ }^{14}$

$60 \%$ of the facilities are understaffed. All the therapists working in these facilities find their work very demanding because they have to handle all the patients alone. If any of the therapists working in these facilities take a sick leave or any emergency leave that means the department will be shut down. Although the government has appointed new staff to all the dental facilities that are understaffed, they have failed to report for duty, this has affected the facilities badly. Most of the patients have to be given appointments because of in adequate staff. A study done in Mangalore, India also stated that the availability of dentists and dental services at health centers were alarmingly low. ${ }^{12}$ Study done in Zambia on health worker shortage suggested that the motivation and commitment of existing health

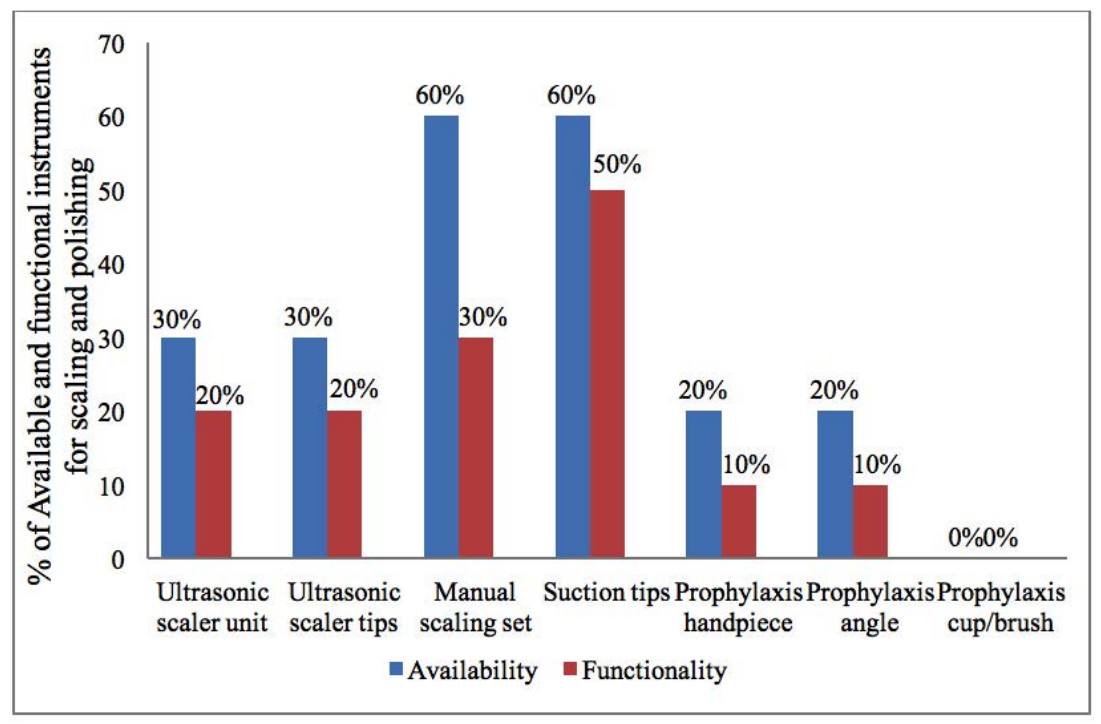

Figure 3. Availability and functionality of instruments for scaling and polishing.

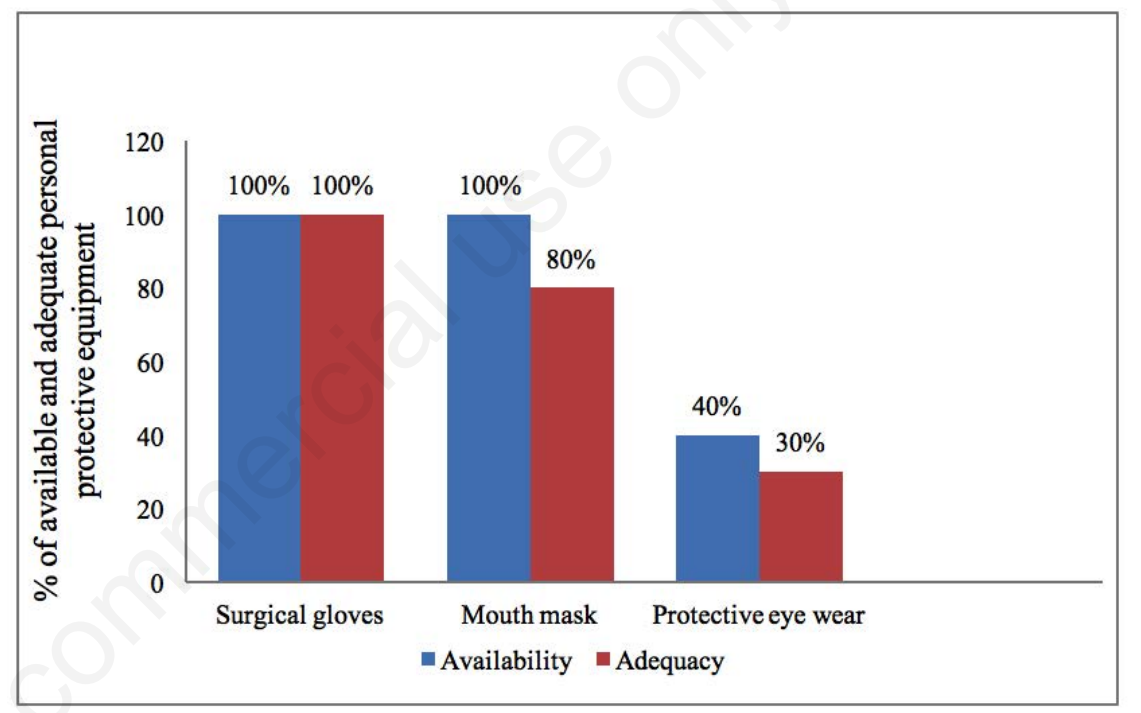

Figure 4. Availability and adequacy of personal protective equipment.

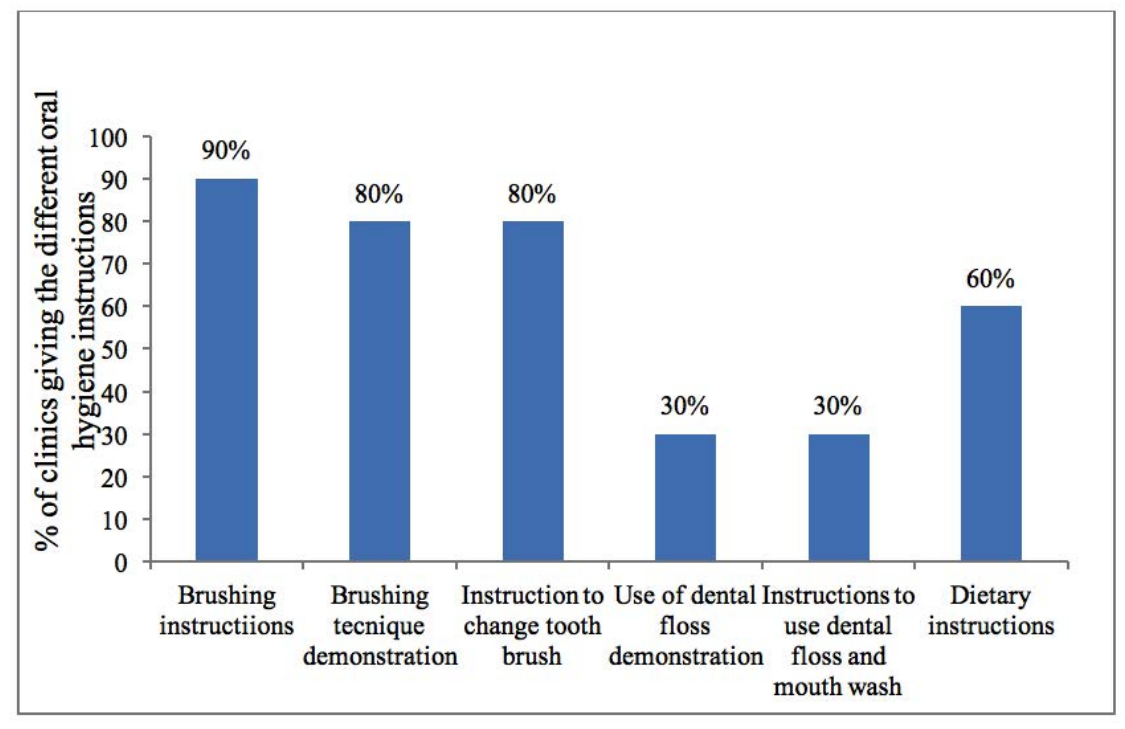

Figure 5. Oral hygiene instructions. 
workers will be enhanced by improving their conditions of service. While increased salaries would be welcomed, they are not the primary motivating factor of health workers. Even with significant gains in training enrolments, retention, graduation, and public sector entry, Zambia is likely to operate with a shortage of human resources for health for at least the next decade unless alternative means of addressing this shortage are introduced. ${ }^{15}$

The DTs are referring a lot of patients to higher facilities for S\&P. But when they were asked whether all these patients were receiving treatment from these higher facilities, their answer was no. Most of the DTs answered that it is not possible for the second level and tertiary hospitals to provide S\&P to all since most of the first level hospitals and all the health centers send the patients who require S\&P to them. Also some patients do not have the finances to go to higher facilities and some do not go due to lack of knowledge about the importance of oral hygiene. In Nigeria it has been found that the oral health care system is not sufficiently responsive to the needs of the populace and there is little stewardship of the system. The system is neither effective nor efficient and the resources available within the system are grossly inadequate and are overstretched in many areas. ${ }^{16}$

\section{Public health significance of the study}

Around the globe many people are suffering from oral pain and other problems of the mouth or teeth. This public health problem is growing rapidly in developing countries where oral health services are limited. ${ }^{17}$ This study helps to bring out the urgent needs of the oral health care system which has to be dealt with in order to provide the basic dental care to the population. It also brings out the difficulties faced by the health workers because of the flaws in the oral health care system.

\section{Conclusions}

Oral health has been highly neglected and there is a great need for providing communities with oral health education and S\&P. Compared to the wide treatment options in the field of dentistry, these public health facilities are only able to provide patients with dental extractions and a few facilities provide temporary dental fillings.

Providing adequate oral hygiene treatment to the general public from the health centers and first level hospitals should be taken into consideration. Improving the working conditions of the DTs must also be taken into consideration. All efforts should be taken by the authorities towards improving and coordinating the oral health care system and importance must be given to promoting oral health education to the populace.

\section{References}

1. World Health Organization. Oral health surveys: basic methods. World Health Organization; 2013.

2. MOH, Republic of Zambia. National Health Policy, 'A Nation of Healthy and Productive People', 2012.

3. Health Professions Council of Zambia. National health standards for Zambia; 2014.

4. World Health Organization and UNAIDS. AIDS epidemic update: WHO Regional Office Europe; 2009.

5. Blaizot A, Vergnes JN, Nuwwareh S, et al. Periodontal diseases and cardiovascular events: meta-analysis of observational studies. Int Dent J 2009;59:197209.

6. Petersen PE. World Health Organization global policy for improvement of oral health-World Health Assembly 2007. Int Dent J 2008;58: 115-21.

7. Chung JH, Hwang HJ, Kim SH, Kim
TH. Associations between Periodontitis and Chronic Obstructive Pulmonary Disease; the 2010-2012 Korean National Health and Nutrition Examination Survey (KNHANES). J Periodontol 2016;0:1-11.

8. Maltz M, Jardim JJ, Alves LS. Health promotion and dental caries. Braz Oral Res 2010;24:18-25.

9. Needleman I, Nibali L, Di Iorio A. Professional mechanical plaque removal for prevention of periodontal diseases in adults--systematic review update. J Clin Periodontol 2015;42: S12-35.

10. Makasa E. The Human Resource crisis in the Zambian Health Sector-a discussion paper. Med J Zambia 2008;35:3.

11. Nyamuryekung'e KK, Lahti SM, Tuominen RJ. The relative patient costs and availability of dental services, materials and equipment in public oral care facilities in Tanzania. BMC Oral Health 2015;15:74.

12. Simon AK, Rao A, Rajesh G, et al. Oral health care availability in health centers of Mangalore taluk, India. Indian J Commun Med 2014;39:218.

13. Vashist A, Parhar S, Gambhir RS, et al. Status of governmental oral health care delivery system in Haryana, India. J Family Med Prim Care 2016;5:547.

14. Ajayi YO, Ajayi EO. Prevalence of ocular injury and the use of protective eye wear among the dental personnel in a teaching hospital. Nig Q J Hosp Med 2008;18:83-6.

15. Gow J, George G, Mutinta G, et al. Health worker shortages in Zambia: An assessment of government responses. J Public Health Policy 2011;32:197-209.

16. Adeniyi AA, Sofola OO, Kalliecharan $\mathrm{RV}$. An appraisal of the oral health care system in Nigeria. Int Dent J 2012;62: 292-300.

17. Petersen PE. Strengthening of oral health systems: oral health through primary health care. Med Principles Pract 2014;23:3-9. 